\title{
Numerical Solution of Singularly Perturbed Differential- Difference Equations with Dual Layer
}

\author{
Lakshmi Sirisha, Y.N. Reddy ${ }^{*}$ \\ Department of Mathematics, National Institute of Technology, WARANGAL, INDIA \\ Corresponding author: ynreddy_nitw@yahoo.com
}

Received September 13, 2014; Revised October 09, 2014; Accepted October 12, 2014

\begin{abstract}
In this paper, we discuss the numerical solution of singularly perturbed differential-difference equations exhibiting dual layer behavior. First the second order singularly perturbed differential-difference equation is replaced by an asymptotically equivalent second order singularly perturbed ordinary differential equation. Then, second order stable central difference scheme has been applied to get a three term recurrence relation which is easily solved by Discrete Invariant Imbedding Algorithm. Some numerical examples have been considered to validate the computational efficiency of the proposed numerical scheme. To analyze the effect of the parameters on the solutions, the numerical solutions have also been plotted using graphs. The error bound and convergence of the method have also been established.
\end{abstract}

Keywords: singular perturbations, differential-difference equations, dual layer, delay parameter, advance parameter

Cite This Article: Lakshmi Sirisha, and Y.N. Reddy, "Numerical Solution of Singularly Perturbed Differential-Difference Equations with Dual Layer." American Journal of Applied Mathematics and Statistics, vol. 2, no. 5 (2014): 336-343. doi: 10.12691/ajams-2-5-7.

\section{Introduction}

Singularly Perturbed Differential-difference equations occur very frequently in mathematical modeling and control theory. More specifically, the boundary value problems (BVPs) for differential-difference equations (DDEs) come up in the study of the effect of time delays in signal transmission of control theory and in the phenomenon where Markov process governs, such as persistence times of population with large number of random fluctuations and time between the impulses of the nerve cell [1,2]. Depending on the parameter values, the resulting solutions of the class of differential-difference equations exhibit oscillations, boundary and interior layers, or turning point behavior. Questions on the existence and uniqueness of the solutions to these BVPs for DDEs were addressed in a series of papers written by Schmidt and coauthors $[3,4]$. Stochastic effects due to neuronal excitation, in neurobiology, were discussed with the help of a DDEs model by Stein [5]. The approximated solutions to this model was given by the same author using Monte Carlo techniques. Tuckwell and Richter [6], Tuckwell and Cope [7] and Wilbur and Rinzel [8] used different methods for obtaining the solutions to Stein's model. Lange and Miura $[9,10,11]$ have also analyzed the layer behavior of DDEs in a series of papers. An extensive numerical work had been initiated by Kadalbajoo and Sharma [12,13].

In this paper, we discuss the numerical solution of singularly perturbed differential-difference equations exhibiting dual layer behavior. First the second order singularly perturbed differential-difference equation is replaced by an asymptotically equivalent second order singularly perturbed ordinary differential equation. Then, second order stable central difference scheme been applied to get a three term recurrence relation which is easily solved by Discrete Invariant Imbedding Algorithm. Some numerical examples have been considered to validate the computational efficiency of the proposed numerical scheme. To analyze the effect of the parameters on the solutions, the numerical solutions have also been plotted using graphs. The error bound and convergence of the method have also been established.

\section{Description of the Method}

Consider singularly perturbed differential difference equation with small delay as well as advance parameters of the form:

$$
\begin{aligned}
& \varepsilon^{2} y^{\prime \prime}(x)+\alpha(x) y(x-\delta)+\omega(x) y(x) \\
& +\beta(x) y(x+\eta)=f(x)
\end{aligned}
$$

$\forall x \in(0,1)$ and subject to the interval and boundary conditions

$$
\begin{array}{ccc}
y(x)=\varphi(x), & \text { on } & -\delta \leq x \leq 0 \\
y(x)=\gamma(x), & \text { on } & 1 \leq x \leq 1+\eta
\end{array}
$$

where $\quad \alpha(x), \quad \beta(x), \quad \omega(x), \quad f(x), \quad \varphi(x)$, and $\gamma(x)$ are bounded and continuously differentiable functions on $(0,1), 0<\varepsilon<<1$ is the singular perturbation parameter; 
and $0<\delta=o(\varepsilon)$ and $0<\eta=o(\varepsilon)$ are the delay and the advance parameters respectively.

By using Taylor series expansion in the neighborhood of the point $x$, we have

$$
\begin{gathered}
y(x-\delta) \approx y(x)-\delta y^{\prime}(x) \\
y(x+\eta) \approx y(x)+\eta y^{\prime}(x)
\end{gathered}
$$

Substituting Eqs. (4) and (5) into Eq. (1), we obtain an asymptotically equivalent singularly perturbed boundary value problem of the form

$$
\begin{gathered}
\varepsilon^{2} y^{\prime \prime}(x)+p(x) y^{\prime}(x)+q(x) y(x)=f(x) \\
y(0)=\varphi(x)=\varphi_{0} \\
y(1)=\gamma(x)=\gamma_{1}
\end{gathered}
$$$$
\text { where } \quad p(x)=\eta \beta(x)-\delta \alpha(x)
$$

$q(x)=\alpha(x)+\beta(x)+\omega(x)$

The transition from Eq. (1) to Eq. (6) is admitted, because of the condition that $0<\delta<<1$ and $0<\eta<<1$ are sufficiently small. Further details on the validity of this transition can be found in Elsgolt's and Norkin [14].

If $\alpha(x)+\beta(x)+\omega(x) \leq 0$ on the interval $[0,1]$, then the solution of Eq. (1) exhibits boundary layers at both ends of the interval $[0,1]$, whereas it exhibits oscillatory behaviour for $\alpha(x)+\beta(x)+\omega(x)>0$. Here, we have considered the first case where the solutions of the problem exhibit dual layers.

Now, we divide the interval $[0,1]$ into $\mathrm{N}$ equal parts with constant mesh length $\mathrm{h}$. Let $0=x_{0}, x_{1}, x_{2}, \ldots, x_{N}=1$ be the mesh points. Then, we have $x_{i}=i h, i=0,1,2, \ldots, N$.

Assuming that $y(x)$ has continuous fourth derivatives on $[0,1]$ and by making use of Taylor's expansion, we obtain

$$
\begin{gathered}
y_{i}^{\prime \prime}=\frac{y_{i+1}-2 y_{i}+y_{i-1}}{h^{2}}-\frac{h^{2}}{12} y_{i}^{(4)}\left(\xi_{1}\right)+R_{1} \\
y_{i}^{\prime}=\frac{y_{i+1}-y_{i-1}}{2 h}-\frac{h^{2}}{6} y_{i}^{\prime \prime \prime}\left(\xi_{2}\right)+R_{2}
\end{gathered}
$$

where $R_{1}=-\frac{2 h^{4} y^{(6)}\left(\xi_{1}\right)}{6 !}$ and $R_{2}=\frac{-h^{4} y^{(5)}\left(\xi_{2}\right)}{5 !}$, for $\xi_{1}, \xi_{2} \in\left[x_{i-1}, x_{i}\right]$.

Using Eqs. (9) and (10) into Eq. (6), we obtain

$$
\begin{aligned}
& \frac{\varepsilon^{2}}{h^{2}}\left[y_{i+1}-2 y_{i}+y_{i-1}\right]+\frac{p_{i}}{2 h}\left[y_{i+1}-y_{i-1}\right] \\
& -\frac{h^{2} p_{i}}{6} y_{i}^{\prime \prime \prime}\left(\xi_{2}\right)+q_{i} y_{i}=f_{i}+R
\end{aligned}
$$

where $\quad R=R_{1}+R_{2}-\frac{\varepsilon^{2} h^{2} y^{(4)}\left(\xi_{1}\right)}{12}$
$p\left(x_{i}\right)=p_{i}, q\left(x_{i}\right)=q_{i}, f\left(x_{i}\right)=f_{i}, y\left(x_{i}\right)=y_{i}$.

Differentiating both sides of Eq. (6), we have and

$$
\varepsilon^{2} y^{\prime \prime \prime}(x)=f^{\prime}-p y^{\prime \prime}-\left(p^{\prime}+q\right) y^{\prime}-q^{\prime} y
$$

By substituting Eq. (12) into Eq. (11) and approximating the converted error term, which has the stabilizing effect, by using Eqs. (9) and (10), we obtain the second order stable central difference scheme as $\varepsilon$ tends to zero.

$$
\begin{aligned}
& {\left[\frac{\varepsilon^{2}}{h^{2}}+\frac{p_{i}^{2}}{6 \varepsilon^{2}}-\frac{p_{i}}{2 h}-\frac{h p_{i}\left(p_{i}^{\prime}+q_{i}\right)}{12 \varepsilon^{2}}\right] y_{i-1}-} \\
& {\left[\frac{2 \varepsilon^{2}}{h^{2}}+\frac{2 p_{i}^{2}}{6 \varepsilon^{2}}-\frac{h^{2} p_{i} q_{i}^{\prime}}{6 \varepsilon^{2}}-q_{i}\right] y_{i}+} \\
& {\left[\frac{\varepsilon^{2}}{h^{2}}+\frac{p_{i}^{2}}{6 \varepsilon^{2}}+\frac{p_{i}}{2 h}+\frac{h p_{i}\left(p_{i}^{\prime}+q_{i}\right)}{12 \varepsilon^{2}}\right] y_{i+1}=} \\
& f_{i}+\frac{h^{2} p_{i}}{6 \varepsilon^{2}} f_{i}^{\prime}
\end{aligned}
$$

Equation (13) can be written as the three term recurrence relation of form:

$$
\begin{aligned}
& E_{i} y_{i-1}-F_{i} y_{i}+G_{i} y_{i+1}=H_{i} ; \\
& i=1,2, \quad \ldots, N-1
\end{aligned}
$$

where

$$
\begin{aligned}
& E_{i}=\frac{\varepsilon^{2}}{h^{2}}+\frac{p_{i}^{2}}{6 \varepsilon^{2}}-\frac{p_{i}}{2 h}-\frac{h p_{i}\left(p_{i}^{\prime}+q_{i}\right)}{12 \varepsilon^{2}} \\
& F_{i}=\frac{2 \varepsilon^{2}}{h^{2}}+\frac{2 p_{i}^{2}}{6 \varepsilon^{2}}-\frac{h^{2} p_{i} q_{i}^{\prime}}{6 \varepsilon^{2}}-q_{i} \\
& G_{i}=\frac{\varepsilon^{2}}{h^{2}}+\frac{p_{i}^{2}}{6 \varepsilon^{2}}+\frac{p_{i}}{2 h}+\frac{h p_{i}\left(p_{i}^{\prime}+q_{i}\right)}{12 \varepsilon^{2}} \\
& H_{i}=f\left(x_{i}\right)+\frac{h^{2} p_{i} f_{i}^{\prime}}{6 \varepsilon^{2}}
\end{aligned}
$$

This gives us the tri-diagonal system which can easily be solved by Thomas Algorithm described in the next section.

\section{Discrete Invariant Imbedding Algorithm}

We give a brief description of discrete invariant imbedding algorithm for solving the tri-diagonal system. Consider the system:

$$
\begin{aligned}
& E_{i} y_{i-1}-F_{i} y_{i}+G_{i} y_{i+1}=H_{i} ; \\
& i=1, \quad 2, \quad \ldots, N-1
\end{aligned}
$$

subject to the boundary conditions

$$
\begin{aligned}
& y_{0}=y(0)=\varphi_{0} ; \\
& y_{N}=y(1)=\gamma_{1}
\end{aligned}
$$

We set

$$
\begin{aligned}
& y_{i}=W_{i} y_{i+1}+T_{i} \text { for } \\
& i=N-1, N-2, \ldots, 2,1
\end{aligned}
$$

where $W_{i}=W\left(x_{i}\right)$ and $T_{i}=T\left(x_{i}\right)$ which are to be determined. 
From (18), we have:

$$
y_{i-1}=W_{i-1} y_{i}+T_{i-1}
$$

By substituting (19) in (15) and comparing with (18) we get the recurrence relations:

$$
\begin{gathered}
W_{i}=\left(\frac{G_{i}}{F_{i}-E_{i} W_{i-1}}\right) \\
T_{i}=\left(\frac{E_{i} T_{i-1}-H_{i}}{F_{i}-E_{i} W_{i-1}}\right)
\end{gathered}
$$

To solve these recurrence relations for $i=1,2, \ldots, N-1$, we need the initial conditions for $W_{0}$ and $T_{0}$. For this we take $y_{0}=\varphi_{0}=W_{0} y_{1}+T_{0}$. We choose $W_{0}=0$ so that the value of $T_{0}=\varphi_{0}$. With these initial values, we compute $W_{i}$ and $T_{i}$ for $i=1,2, \ldots N-1$ from (20) and (21) in forward process, and then obtain $y_{i}$ in the backward process from (17) and (18).

\section{Stability and Convergence Analysis}

In this section we discuss the error analysis of the method. Writing the tri-diagonal system (14) in matrixvector form, we get

$$
A Y=C
$$

where, $A=\left(m_{i j}\right), 1 \leq i, j \leq N-1 \quad$ is $\quad$ a tri-diagonal matrix of order $N-1$, with

$$
\begin{aligned}
& m_{i i+1}=-\varepsilon^{2}+h\left[-\frac{p_{i}}{2}\right]+h^{2}\left[-\frac{p_{i}^{2}}{6 \varepsilon^{2}}\right]+ \\
& h^{3}\left[-\frac{p_{i}\left(p_{i}^{\prime}+q_{i}\right)}{12 \varepsilon^{2}}\right] \\
& m_{i i}=2 \varepsilon^{2}+h^{2}\left[\frac{p_{i}^{2}}{3 \varepsilon^{2}}-q_{i}\right]+h^{4}\left(-\frac{p_{i} q_{i}^{\prime}}{6 \varepsilon^{2}}\right) \\
& m_{i i-1}=-\varepsilon^{2}+h\left[\frac{p_{i}}{2}\right]+h^{2}\left[-\frac{p_{i}^{2}}{6 \varepsilon^{2}}\right]+ \\
& h^{3}\left[\frac{p_{i}\left(p_{i}^{\prime}+q_{i}\right)}{2 \varepsilon^{2}}\right]
\end{aligned}
$$

and $C=\left(d_{i}\right)$ is a column vector with $d_{\mathrm{i}}=-h^{2}\left(f\left(x_{i}\right)+\frac{h^{2} p_{i} f_{i}^{\prime}}{6 \varepsilon^{2}}\right)$, where $i=1,2, \ldots, N-1 \quad$ with local truncation error

$$
T_{\mathrm{i}}(h)=\frac{h^{4}}{2} K+o\left(h^{5}\right)
$$

where

$$
K=\left(-a_{i} \delta+b_{i} \eta\right) y_{i}^{\prime \prime \prime}\left(\xi_{2}\right)+\left(\varepsilon^{2}+\frac{\delta^{2}}{2}+\frac{\eta^{2}}{2}\right) y_{i}^{(4)}\left(\xi_{1}\right)
$$

We also have

$$
\mathrm{A} \overline{\mathrm{Y}}-T(h)=C
$$

where $\bar{Y}=\left(\overline{y_{0}}, \overline{y_{1}}, \overline{y_{2}}, \ldots, \overline{y_{N}}\right)^{T}$ denotes the actual solution and $T(h)=\left(T_{1}(h), T_{2}(h), \ldots, T_{N}(h)\right)^{T} \quad$ is the local truncation error.

From Eqs. (22) and (24), we get

$$
A(\bar{Y}-Y)=T(h)
$$

Thus, we obtain the error equation

$$
A E=T(h)
$$

where $E=\bar{Y}-Y=\left(e_{0}, e_{1}, e_{2}, \ldots, e_{N}\right)^{T}$.

Let $S_{i}$ be the sum of elements of the $i^{\text {th }}$ row of A, then we have

$$
\begin{aligned}
& S_{i}=\varepsilon^{2}+h\left(-\frac{p_{i}}{2}\right)+h^{2}\left(\frac{-p_{i}}{2 h}+\frac{p_{i}^{2}}{3 \varepsilon^{2}}-q_{i}\right)+O\left(h^{3}\right) \text { for } \mathrm{i}=1 \\
& S_{i}=h^{2}\left(\frac{p^{2}}{3 \varepsilon^{2}}-q_{i}\right)+O\left(h^{3}\right) \text { for } \mathrm{i}=2,3, . . \mathrm{N}-2 \\
& S_{i}=\varepsilon^{2}+h\left(\frac{p_{i}}{2}\right)+h^{2}\left(-\frac{p_{i}}{6 \varepsilon^{2}}+\frac{p_{i}^{2}}{3 \varepsilon^{2}}-q_{i}\right)+O\left(h^{3}\right)
\end{aligned}
$$

for $\mathrm{i}=\mathrm{N}-1$

and $C=\left(d_{i}\right)$ is a column vector with $d_{\mathrm{i}}=f\left(x_{i}\right)+\frac{h^{2} p_{i} f_{i}^{\prime}}{6 \varepsilon^{2}}$, where $i=1,2 \ldots N-1$ with local truncation error

$$
T_{\mathrm{i}}(h)=\frac{h^{4}}{2}\left[\begin{array}{l}
\left(-a_{i} \delta+b_{i} \eta\right) y_{i}^{\prime \prime \prime}\left(\xi_{2}\right) \\
+\left(\varepsilon^{2}+\frac{\delta^{2}}{2}+\frac{\eta^{2}}{2}\right) y_{i}^{(4)}\left(\xi_{1}\right)
\end{array}\right]+o\left(h^{5}\right)
$$

Since $0<\varepsilon<<1$ and $\delta=o(\varepsilon)$, for sufficiently small $h$ the matrix $A$ is irreducible and monotone (Mohanty and Jha [15]). Then it follows that $A^{-1}$ exists and its elements are non negative.

Hence, from Eq. (26), we get

$$
E=A^{-1} T(h)
$$

and

$$
\|E\| \leq\left\|A^{-1}\right\| \cdot\|T(h)\|
$$

Let $\bar{m}_{k, i}$ be the $(k, i)^{t h}$ element of $A^{-1}$. Since $\bar{m}_{k, i} \geq 0$, from the theory of matrices we have

$$
\sum_{i=1}^{N-1} \bar{m}_{\mathrm{k}, \mathrm{i}} S_{\mathrm{i}}=1, k=1,2, \ldots N-1
$$


Therefore,

$$
\sum_{i=1}^{N-1} \bar{m}_{k, i} \leq \frac{1}{\min _{1 \leq i \leq N-1} S_{i}}=\frac{1}{h^{2}\left|B_{i_{0}}\right|}
$$

where $B_{i_{0}}=\frac{p_{i}^{2}}{3 \varepsilon^{2}}-q_{i}$

We define $\quad\left\|A^{-1}\right\|=\max _{1 \leq k \leq N-1} \sum_{i=1}^{N-1}\left|\bar{m}_{k, i}\right|$ and

$\|T(h)\|=\max _{1 \leq i \leq N-1}\left|T_{i}(h)\right|$.

From (23), (27), (28) and (30), we get

$$
e_{j}=\sum_{i=1}^{N-1} \bar{m}_{k . i} T_{\mathrm{i}}(h), \quad j=1,2,3 . . N-1
$$

which implies

$$
e_{j} \leq \frac{h^{4} K}{h^{2}\left|B_{i_{0}}\right|}=\frac{\mathrm{h}^{2} K}{\left|B_{i_{0}}\right|}, j=1,2 \ldots N-1
$$

where $i_{0}$ is some number between $i$ and $N$.

Therefore, $\|E\|=o\left(h^{2}\right)$.

Hence, our method gives a quadratic order convergence for uniform mesh.

Further, as already mentioned, because of substituting Eq. (12) into Eq. (11) and approximating the converted error term, which has the stabilizing effect, by using Eqs. (9) and (10), we have obtained the second order stable central difference scheme as $\varepsilon$ tends to zero. Regarding the convergence and dependency of the scheme on inverse power of $\varepsilon$ is yet be investigated in future works.

\section{Numerical Examples}

To demonstrate the applicability of the method, we have applied it to two cases of the problems of the form:

$$
\begin{aligned}
& \varepsilon^{2} y^{\prime \prime}(x)+\alpha(x) y(x-\delta)+\omega(x) y(x) \\
& +\beta(x) y(x+\eta)=f(x)
\end{aligned}
$$

$\forall x \in(0,1)$ and subject to the interval and boundary conditions

$$
\begin{array}{lll}
y(x)=\varphi(x), & \text { on } & -\delta \leq x \leq 0 \\
y(x)=\gamma(x), & \text { on } & 1 \leq x \leq 1+\eta
\end{array}
$$

The exact solution of such boundary value problems having constant coefficients (i.e. $\alpha(x)=\alpha$, $\beta(x)=\beta, \quad \omega(x)=\omega, \quad f(x)=f, \quad \varphi(x)=\varphi$ and $\gamma(x)=\gamma$ are constants) is given by:

$$
\begin{aligned}
& y(x)=\left(\begin{array}{l}
{\left[(1-\alpha-\beta-\omega) \exp \left(m_{2}\right)-1\right] \exp \left(m_{1} x\right)-} \\
{\left[(1-\alpha-\beta-\omega) \exp \left(m_{1}\right)\right] \exp \left(m_{2} x\right)}
\end{array}\right) / \\
& {\left[(\alpha+\beta+\omega)\left(\exp \left(m_{1}\right)-\exp \left(m_{2}\right)\right)\right]+1 /(\alpha+\beta+\omega)}
\end{aligned}
$$

where

$$
\begin{aligned}
& m_{1}=\frac{\left[(\alpha \delta-\beta \eta)+\sqrt{(\beta \eta-\alpha \delta)^{2}-4 \varepsilon^{2}(\alpha+\beta+\omega)}\right]}{2 \varepsilon^{2}} \\
& m_{2}=\frac{\left[(\alpha \delta-\beta \eta)-\sqrt{(\beta \eta-\alpha \delta)^{2}-4 \varepsilon^{2}(\alpha+\beta+\omega)}\right]}{2 \varepsilon^{2}}
\end{aligned}
$$

Example 1: Consider the model problem given by equations (1)-(3) with

$$
\begin{aligned}
& \alpha(x)=-2, \quad \beta(x)=-2, \quad \omega(x)=-1, \quad f(x)=1, \\
& \varphi(x)=1, \quad \gamma(x)=0 .
\end{aligned}
$$

The exact solution of the problem is given by Eq. (32). The numerical results are given in Table 1- Table 4 for

$\varepsilon=0.1,0.01$ and different values of the delay and advance parameters.

Example 2: Consider the model problem given by equations (1)-(3) with

$\alpha(x)=0.25, \quad \beta(x)=0.25, \quad \omega(x)=-1, f(x)=1$,

$\varphi(x)=1, \quad \gamma(x)=0$.

The exact solution of the problem is given by Eq. (37). The numerical results are given in Table 5 - Table 9 for

$\varepsilon=0.1,0.01$ and different values of the delay and advance parameters.

\section{Discussion and Conclusion}

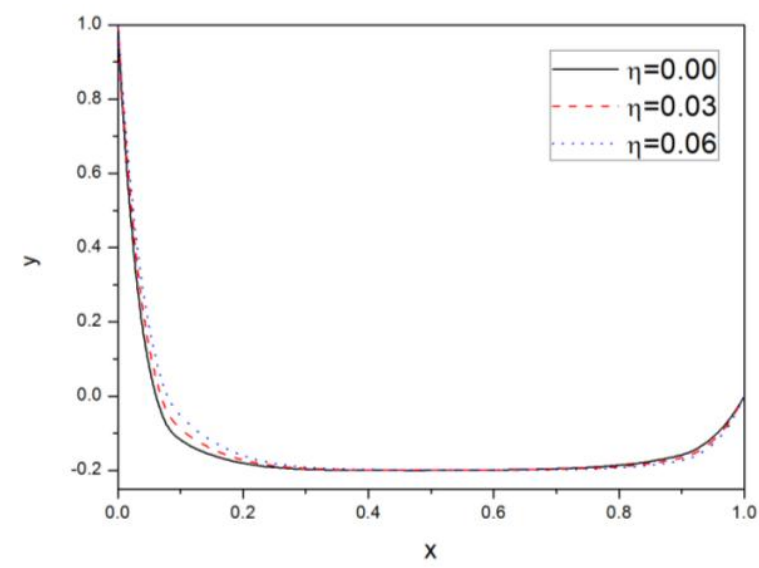

Figure 1. Numerical solution of Example 1 for $\varepsilon=0.1$ and $\delta=0.07$

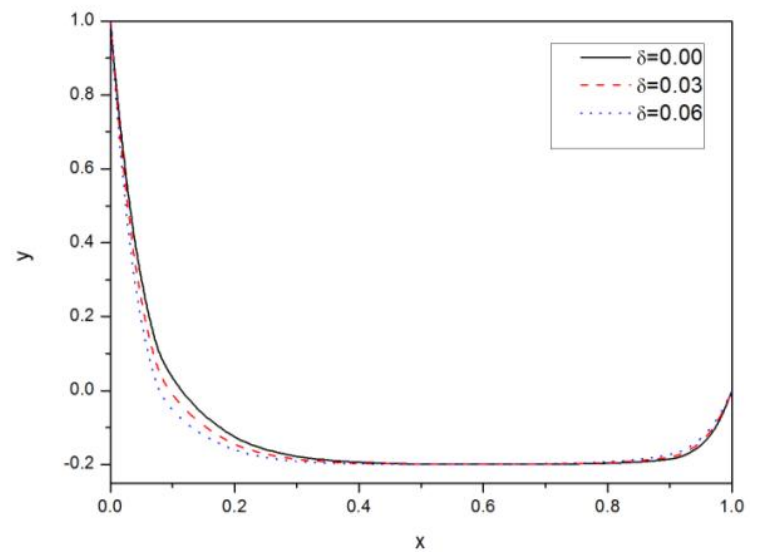

Figure 2. Numerical solution of Example 1 for $\varepsilon=0.1$ and $\eta=0.05$ 


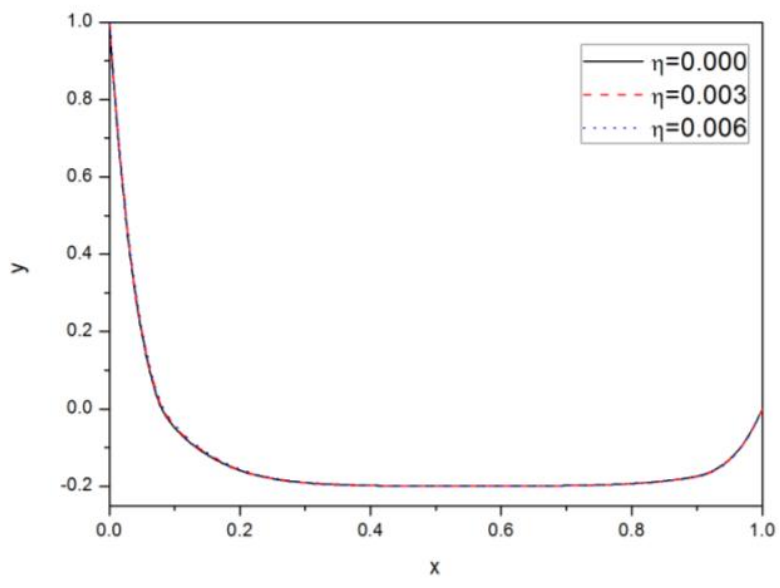

Figure 3. Numerical solution of Example 1 for $\varepsilon=0.1$ and $\delta=0.007$

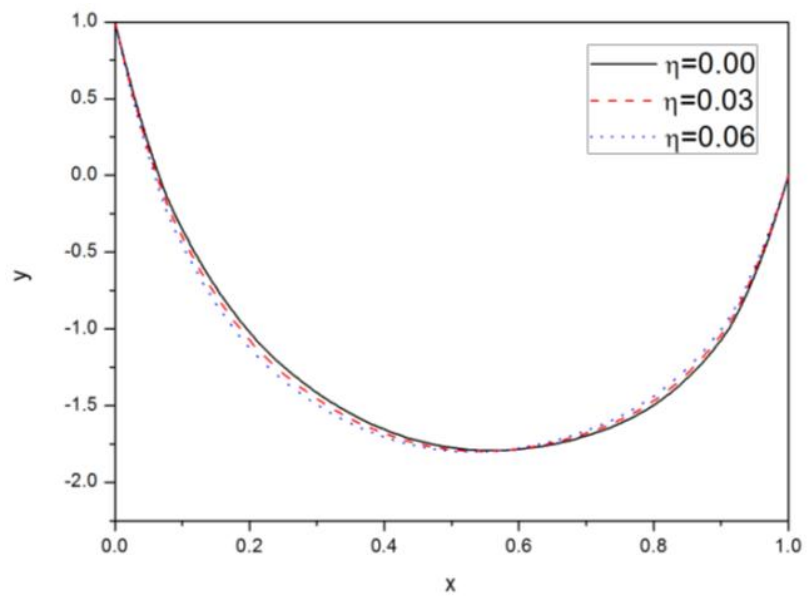

Figure 4. Numerical solution of Example 2 for $\varepsilon=0.1$ and $\delta=0.05$

In this paper, we discuss the numerical solution of singularly perturbed differential-difference equations exhibiting dual layer behavior. First the second order singularly perturbed differential-difference equation is replaced by an asymptotically equivalent second order singularly perturbed ordinary differential equation. Then, second order stable central difference scheme been applied to get a three term recurrence relation which is easily solved by Discrete Invariant Imbedding Algorithm. The error bound and convergence of the method have also been established. To demonstrate the applicability of the method, we applied it on two model examples for different values of the perturbation, delay and advance parameters. The numerical solutions are presented in tables and compared with the exact solution. To analyze the effect of the parameters on the solutions, the numerical solutions have also been plotted using graphs. From the results presented in tables, it is observed that the present method approximates the exact solution very well. For the examples we have considered (i.e., examples in which both the negative and positive shifts are non zero), it is observed that increasing the value of either $\delta$ or $\eta$, the thickness of the left boundary layer decreases and that of the right boundary layer increases. Thus, it can be concluded that a negative shift is more dominant than a positive shift. The error bound and convergence of the method have also been established and it is observed that our method gives second order convergence.

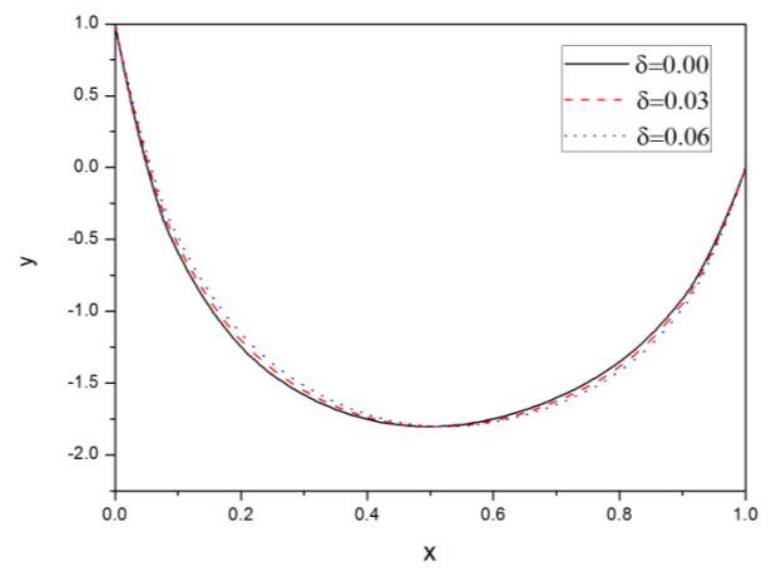

Figure 5. Numerical solution of Example 2 for $\varepsilon=0.1$ and $\eta=0.07$

Table 1. Numerical solution of Example 1 for $\varepsilon=0.01$ and $\eta=0.005$

\begin{tabular}{|c|c|c|c|c|c|c|}
\hline \multirow{2}{*}{$x$} & \multicolumn{2}{|c|}{$\eta=0.00$} & \multicolumn{2}{c}{$\eta=0.03$} & \multicolumn{2}{c|}{$\eta=0.06$} \\
\cline { 2 - 7 } & Num. Sol & Exact Sol. & Num. Sol & Exact Sol. & Num. Sol & Exact Sol. \\
\hline 0.00 & 1.0000000 & 1.0000000 & 1.0000000 & 1.0000000 & 1.0000000 & 1.0000000 \\
0.01 & 0.6865117 & 0.6851608 & 0.7195210 & 0.7186640 & 0.7503216 & 0.7497953 \\
0.03 & 0.2838279 & 0.2816195 & 0.3399114 & 0.3384032 & 0.3960042 & 0.3950146 \\
0.05 & 0.0640568 & 0.0620510 & 0.1170176 & 0.1155430 & 0.1737903 & 0.1727564 \\
0.07 & -0.0558867 & -0.0574169 & -0.0138580 & -0.0150690 & 0.0344265 & 0.0335193 \\
0.09 & -0.1213478 & -0.1224199 & -0.0907037 & -0.0916171 & -0.0529769 & -0.0537080 \\
0.20 & -0.1971861 & -0.1972706 & -0.1941548 & -0.1942628 & -0.1887028 & -0.1888273 \\
0.40 & -0.1999828 & -0.1999833 & -0.19996888 & -0.1999699 & -0.1998930 & -0.1998954 \\
0.60 & -0.1997188 & -0.1997202 & -0.1998867 & -0.1998877 & -0.1999598 & -0.1999604 \\
0.80 & -0.1925013 & -0.1925204 & -0.1952428 & -0.1952640 & -0.1972004 & -0.1972220 \\
0.90 & -0.1612736 & -0.1613231 & -0.1691547 & -0.1692234 & -0.1763377 & -0.1764290 \\
0.92 & -0.1462211 & -0.1462761 & -0.1551712 & -0.1552511 & -0.1637380 & -0.1638500 \\
0.94 & -0.1253180 & -0.1253752 & -0.1348484 & -0.1349354 & -0.1444293 & -0.1445581 \\
0.96 & -0.0962900 & -0.0963429 & -0.1053123 & -0.1053967 & -0.1148390 & -0.1149706 \\
0.98 & -0.0559791 & -0.0560159 & -0.0623863 & -0.0624476 & -0.0694925 & -0.0695934 \\
1.00 & 0.0000000 & 0.0000000 & 0.0000000 & 0.0000000 & 0.0000000 & 0.0000000 \\
\hline
\end{tabular}


Table 2. Numerical solution of Example 1 for $\varepsilon=0.1$ and $\eta=0.05$

\begin{tabular}{|c|c|c|c|c|c|c|}
\hline \multirow{2}{*}{$x$} & \multicolumn{2}{|c|}{$\delta=0.00$} & \multicolumn{2}{|c|}{$\delta=0.03$} & \multicolumn{2}{|c|}{$\delta=0.06$} \\
\hline & Num. Sol & Exact Sol. & Num. Sol & Exact Sol. & Num. Sol & Exact Sol. \\
\hline 0.00 & 1.0000000 & 1.0000000 & 1.0000000 & 1.0000000 & 1.0000000 & 1.0000000 \\
\hline 0.01 & 0.8033835 & 0.8031978 & 0.7783813 & 0.7780662 & 0.7503216 & 0.7497953 \\
\hline 0.03 & 0.5015174 & 0.5011280 & 0.4503721 & 0.4497440 & 0.3960042 & 0.3950146 \\
\hline 0.05 & 0.2904671 & 0.2900134 & 0.2323303 & 0.2316346 & 0.1737903 & 0.1727564 \\
\hline 0.07 & 0.1429109 & 0.1424670 & 0.0873886 & 0.0867413 & 0.0344265 & 0.0335193 \\
\hline 0.09 & 0.0397468 & 0.0393478 & -0.0089604 & -0.0095134 & -0.0529769 & -0.0537080 \\
\hline 0.20 & -0.166511 & -0.1666352 & -0.1797828 & -0.1799126 & -0.1887028 & -0.1888273 \\
\hline 0.40 & -0.1990654 & -0.1990723 & -0.1996593 & -0.1996636 & -0.1998930 & -0.1998954 \\
\hline 0.60 & -0.1999709 & -0.1999713 & -0.1999826 & -0.1999830 & -0.1999598 & -0.1999604 \\
\hline 0.80 & -0.1992299 & -0.1992467 & -0.1984755 & -0.1984956 & -0.1972004 & -0.1972220 \\
\hline 0.90 & -0.1875954 & -0.1877314 & -0.1825396 & -0.1826546 & -0.1763377 & -0.1764290 \\
\hline 0.92 & -0.1783693 & -0.1785593 & -0.1715653 & -0.1717152 & -0.1637380 & -0.1638500 \\
\hline 0.94 & -0.1622811 & -0.1625297 & -0.1536933 & -0.1538765 & -0.1444293 & -0.1445581 \\
\hline 0.96 & -0.1342267 & -0.1345162 & -0.1245882 & -0.1247873 & -0.1148390 & -0.1149706 \\
\hline 0.98 & -0.0853063 & -0.08555893 & -0.0771897 & -0.0773519 & -0.0694925 & -0.0695934 \\
\hline 1.00 & 0.0000000 & 0.0000000 & 0.0000000 & 0.0000000 & 0.0000000 & 0.0000000 \\
\hline
\end{tabular}

Table 3. Numerical solution of Example 1 for $\varepsilon=0.01$ and $\eta=0.005$

\begin{tabular}{|c|c|c|c|c|c|c|}
\hline \multirow{2}{*}{$x$} & \multicolumn{2}{|c|}{$\delta=0.000$} & \multicolumn{2}{|c|}{$\delta=0.003$} & \multicolumn{2}{|c|}{$\delta=0.006$} \\
\hline & Num. Sol & Exact Sol. & Num. Sol & Exact Sol. & Num. Sol & Exact Sol. \\
\hline 0.00 & 1.0000000 & 1.0000000 & 1.0000000 & 1.0000000 & 1.0000000 & 1.0000000 \\
\hline 0.01 & 0.0099730 & 9.4346596 & -0.0156722 & -0.0447429 & -0.0269678 & -0.0842104 \\
\hline 0.03 & -0.1935712 & -0.1944365 & -0.1956507 & -0.1974010 & -0.1964023 & -0.1989219 \\
\hline 0.05 & -0.1998031 & -0.1998453 & -0.1998973 & -0.1999564 & -0.1999251 & -0.1999899 \\
\hline 0.07 & -0.1999939 & -0.1999956 & -0.1999975 & -0.1999992 & -0.1999984 & -0.1999999 \\
\hline 0.09 & -0.1999998 & -0.1999998 & -0.1999999 & -0.1999999 & -0.1999999 & -0.1999999 \\
\hline 0.20 & -0.19999999 & -0.2000000 & -0.2000000 & -0.2000000 & -0.2000000 & -0.2000000 \\
\hline 0.40 & -0.2000000 & -0.2000000 & -0.2000000 & -0.2000000 & -0.2000000 & -0.2000000 \\
\hline 0.60 & -0.20000000 & -0.2000000 & -0.2000000 & -0.2000000 & -0.2000000 & -0.2000000 \\
\hline 0.80 & -0.2000000 & -0.2000000 & -0.2000000 & -0.2000000 & -0.2000000 & -0.2000000 \\
\hline 0.90 & -0.1999999 & -0.1999999 & -0.1999999 & -0.1999999 & -0.1999999 & -0.1999999 \\
\hline 0.92 & -0.1999999 & -0.1999999 & -0.1999999 & -0.1999999 & -0.1999999 & -0.1999999 \\
\hline 0.94 & -0.1999975 & -0.1999999 & -0.1999982 & -0.1999999 & -0.1999978 & -0.1999994 \\
\hline 0.96 & -0.1998942 & -0.1999971 & -0.1999141 & -0.1999886 & -0.1999012 & -0.1999614 \\
\hline 0.98 & 1.4414729 & 1.4520547 & -0.1958559 & -0.1984956 & -0.1955569 & -0.1972220 \\
\hline 1.00 & 0.0000000 & 0.0000000 & 0.0000000 & 0.0000000 & 0.0000000 & 0.0000000 \\
\hline
\end{tabular}

Table 4. Numerical solution of Example 1 for $\varepsilon=0.01$ and $\delta=0.007$

\begin{tabular}{|c|c|c|c|c|c|c|}
\hline \multirow{2}{*}{$x$} & \multicolumn{2}{|c|}{$\eta=0.000$} & \multicolumn{2}{|c|}{$\eta=0.003$} & \multicolumn{2}{|c|}{$\eta=0.006$} \\
\hline & Num. Sol & Exact Sol. & Num. Sol & Exact Sol. & Num. Sol & Exact Sol. \\
\hline 0.00 & 1.0000000 & 1.0000000 & 1.0000000 & 1.0000000 & 1.0000000 & 1.0000000 \\
\hline 0.01 & 0.7532580 & 0.7527575 & 0.7561664 & 0.7556906 & 0.7590463 & 0.7585941 \\
\hline 0.03 & 0.4015461 & 0.4005991 & 0.4070688 & 0.4061631 & 0.4125708 & 0.4117047 \\
\hline 0.05 & 0.1796010 & 0.1786056 & 0.1854273 & 0.1844693 & 0.1912668 & 0.1903452 \\
\hline 0.07 & 0.0395443 & 0.0386653 & 0.0447073 & 0.0438562 & 0.0499135 & 0.0490898 \\
\hline 0.09 & -0.0488374 & -0.0495501 & -0.0446356 & -0.0453300 & -0.0403729 & -0.0410490 \\
\hline 0.20 & -0.1879838 & -0.1881093 & -0.1872289 & -0.1873554 & -0.1864372 & -0.1865645 \\
\hline 0.40 & -0.1998792 & -0.1998817 & -0.1998636 & -0.1998663 & -0.1998463 & -0.1998492 \\
\hline 0.60 & -0.1999638 & -0.1999644 & -0.1999674 & -0.1999680 & -0.1999706 & -0.1999711 \\
\hline 0.80 & -0.1973563 & -0.1973778 & -0.1975054 & -0.1975269 & -0.1976480 & -0.1976693 \\
\hline 0.90 & -0.1770059 & -0.1770996 & -0.1776639 & -0.1777600 & -0.1783116 & -0.1784100 \\
\hline 0.92 & -0.1645595 & -0.1646751 & -0.1653733 & -0.1654924 & -0.1661788 & -0.1663017 \\
\hline 0.94 & -0.1453762 & -0.1455098 & -0.1463195 & -0.1464582 & -0.1472590 & -0.1474027 \\
\hline 0.96 & -0.1158092 & -0.1159466 & -0.1167813 & -0.1169247 & -0.1177551 & -0.1179046 \\
\hline 0.98 & -0.07023810 & -0.07034401 & -0.0709894 & -0.0711006 & -0.0717464 & -0.07186309 \\
\hline 1.00 & 0.0000000 & 0.0000000 & 0.0000000 & 0.0000000 & 0.0000000 & 0.0000000 \\
\hline
\end{tabular}


Table 5. Numerical solution of Example 2 for $\varepsilon=0.1$ and $\delta=0.07$

\begin{tabular}{|c|c|c|c|c|c|c|}
\hline \multirow{2}{*}{$x$} & \multicolumn{2}{|c|}{$\eta=0.00$} & \multicolumn{2}{c|}{$\eta=0.03$} & \multicolumn{2}{c|}{$\eta=0.06$} \\
\cline { 2 - 7 } & Num. Sol & Exact Sol. & Num. Sol & Exact Sol. & Num. Sol & Exact Sol. \\
\hline 0.00 & 1.0000000 & 0.9999999 & 1.0000000 & 1.0000000 & 1.0000000 & 1.0000000 \\
0.01 & 0.8183607 & 0.8183354 & 0.8088846 & 0.8088533 & 0.7989101 & 0.7988714 \\
0.03 & 0.4874503 & 0.4873831 & 0.4624642 & 0.4623817 & 0.4363524 & 0.4362513 \\
0.05 & 0.1954543 & 0.1953554 & 0.1588579 & 0.1587372 & 0.1208889 & 0.1207420 \\
0.07 & -0.0621927 & -0.0623151 & -0.1072107 & -0.1073590 & -0.1535774 & -0.1537566 \\
0.09 & -0.2895191 & -0.2896583 & -0.3403657 & -0.3405333 & -0.3923514 & -0.3925523 \\
0.20 & -1.1372046 & -1.1373637 & -1.1922623 & -1.1924467 & -1.2462042 & -1.2464166 \\
0.40 & -1.7372768 & -1.7373880 & -1.7638784 & -1.7639980 & -1.7870329 & -1.7871602 \\
0.60 & -1.8480587 & -1.8481741 & -1.8464250 & -1.8465377 & -1.8412441 & -1.8413530 \\
0.80 & -1.5769603 & -1.5771666 & -1.5468993 & -1.5470824 & -1.5148317 & -1.5149930 \\
0.90 & -1.0929054 & -1.0931264 & -1.0573817 & -1.0575696 & -1.0216905 & -1.0218494 \\
0.92 & -0.9387154 & -0.9389219 & -0.9052246 & -0.9053988 & -0.8719102 & -0.8720563 \\
0.94 & -0.7574103 & -0.7575915 & -0.7278532 & -0.7280047 & -0.6987333 & -0.6988593 \\
0.96 & -0.5443304 & -0.5444717 & -0.5211709 & -0.5212881 & -0.4985660 & -0.4986627 \\
0.98 & -0.2940026 & -0.2940853 & -0.2804057 & -0.2804737 & -0.2672547 & -0.2673104 \\
1.00 & 0.0000000 & 0.0000000 & 0.0000000 & 0.0000000 & 0.0000000 & 0.0000000 \\
\hline
\end{tabular}

Table 6. Numerical solution of Example 2 for $\varepsilon=0.1$ and $\delta=0.05$

\begin{tabular}{|c|c|c|c|c|c|c|}
\hline \multirow{2}{*}{$x$} & \multicolumn{2}{|c|}{$\eta=0.00$} & \multicolumn{2}{c|}{$\eta=0.03$} & \multicolumn{2}{c|}{$\eta=0.06$} \\
\cline { 2 - 7 } & Num. Sol & Exact Sol. & Num. Sol & Exact Sol. & Num. Sol & Exact Sol. \\
\hline 0.00 & 1.0000000 & 1.0000000 & 1.0000000 & 1.0000000 & 1.0000000 & 1.00000000 \\
0.01 & 0.8120990 & 0.8120698 & 0.8022899 & 0.8022538 & 0.8022899 & 0.8022538 \\
0.03 & 0.4709201 & 0.4708430 & 0.4451785 & 0.4450840 & 0.4451785 & 0.4450840 \\
0.05 & 0.1712142 & 0.1711012 & 0.1336911 & 0.1335535 & 0.1336911 & 0.1335535 \\
0.07 & -0.0920465 & -0.0921858 & -0.1379828 & -0.1381512 & -0.1379828 & -0.1381512 \\
0.09 & -0.3232787 & -0.3234363 & -0.3749112 & -0.3751004 & -0.3749112 & -0.3751004 \\
0.20 & -1.1740124 & -1.1741881 & -1.2283744 & -1.2285771 & -1.2283744 & -1.2285771 \\
0.40 & -1.7553832 & -1.7555000 & -1.7797106 & -1.7798354 & -1.7797106 & -1.7798354 \\
0.60 & -1.8473675 & -1.8474813 & -1.8433593 & -1.8434697 & -1.8433593 & -1.8434697 \\
0.80 & -1.5571593 & -1.5573501 & -1.5257225 & -1.5258910 & -1.5257225 & -1.5258910 \\
0.90 & -1.0692553 & -1.0694538 & -1.0335891 & -1.0337572 & -1.0335891 & -1.0337572 \\
0.92 & -0.9163802 & -0.9165646 & -0.8829813 & -0.8831362 & -0.8829813 & -0.8831362 \\
0.94 & -0.7376657 & -0.7378266 & -0.7083807 & -0.7085147 & -0.7083807 & -0.7085147 \\
0.96 & -0.5288348 & -0.5289596 & -0.5060325 & -0.5061356 & -0.5060325 & -0.5061356 \\
0.98 & -0.2848910 & -0.2849636 & -0.2715858 & -0.2716453 & -0.2715858 & -0.2716453 \\
1.00 & 0.0000000 & 0.0000000 & 0.0000000 & 0.0000000 & 0.0000000 & 0.0000000 \\
\hline
\end{tabular}

Table 7. Numerical solution of Example 2 for $\varepsilon=0.1$ and $\eta=0.07$

\begin{tabular}{|c|c|c|c|c|c|c|}
\hline \multirow{2}{*}{$x$} & \multicolumn{2}{|c|}{$\delta=0.00$} & \multicolumn{2}{c|}{$\delta=0.03$} & \multicolumn{2}{c|}{$\delta=0.06$} \\
\cline { 2 - 7 } & Num. Sol & Exact Sol. & Num. Sol & Exact Sol. & Num. Sol & Exact Sol. \\
\hline 0.00 & 1.0000000 & 1.0000000 & 1.0000000 & 1.0000000 & 1.0000000 & 1.0000000 \\
0.01 & 0.7699612 & 0.7698946 & 0.78120489 & 0.7811503 & 0.79198757 & 0.7919431 \\
0.03 & 0.3616734 & 0.3615028 & 0.39048232 & 0.3903416 & 0.41834448 & 0.4182289 \\
0.05 & 0.0138906 & 0.0136479 & 0.05488402 & 0.0546822 & 0.09486995 & 0.0947029 \\
0.07 & -0.2823103 & -0.2826001 & -0.2333342 & -0.2335772 & -0.1851474 & -0.1853502 \\
0.09 & -0.5345307 & -0.5348488 & -0.4808240 & -0.4810930 & -0.4275176 & -0.4277438 \\
0.20 & -1.3813217 & -1.3816204 & -1.3324517 & -1.3327162 & -1.2813311 & -1.2815636 \\
0.40 & -1.8307168 & -1.8308588 & -1.8174851 & -1.8176227 & -1.8004590 & -1.8005908 \\
0.60 & -1.8111612 & -1.8112550 & -1.8250705 & -1.8251706 & -1.8358744 & -1.8359801 \\
0.80 & -1.4221891 & -1.4223001 & -1.4578803 & -1.4580088 & -1.4925106 & -1.4926583 \\
0.90 & -0.9276793 & -0.9277782 & -0.9625572 & -0.9626758 & -0.9979376 & -0.9980792 \\
0.92 & -0.7855529 & -0.7856421 & -0.8173726 & -0.8174803 & -0.8499095 & -0.8500390 \\
0.94 & -0.6244124 & -0.6244879 & -0.6516136 & -0.6517054 & -0.6796459 & -0.6797571 \\
0.96 & -0.4417402 & -0.4417970 & -0.4624009 & -0.4624704 & -0.4838567 & -0.4839416 \\
0.98 & -0.2346815 & -0.2347136 & -0.2464471 & -0.2464867 & -0.2587582 & -0.2588068 \\
1.00 & 0.0000000 & 0.0000000 & 0.0000000 & 0.0000000 & 0.0000000 & 0.0000000 \\
\hline
\end{tabular}


Table 8. Numerical solution of Example 2 for $\varepsilon=0.01$ and $\eta=0.007$

\begin{tabular}{|c|c|c|c|c|c|c|}
\hline \multirow{2}{*}{$x$} & \multicolumn{2}{|c|}{$\delta=0.000$} & \multicolumn{2}{|c|}{$\delta=0.003$} & \multicolumn{2}{|c|}{$\delta=0.006$} \\
\hline & Num. Sol & Exact Sol. & Num. Sol & Exact Sol. & Num. Sol & Exact Sol. \\
\hline 0.00 & 1.0000000 & 1.0000000 & 1.0000000 & 1.0000000 & 1.0000000 & 1.0000000 \\
\hline 0.01 & 0.6202934 & 0.6520131 & 0.5688404 & 0.5954177 & -0.5171991 & -0.5393302 \\
\hline 0.03 & 1.7081782 & 1.7278461 & 1.6742971 & 1.6921075 & -1.6377519 & -1.6537310 \\
\hline 0.05 & 1.9382767 & 1.9450530 & 1.9258766 & 1.9325082 & -1.9115028 & -1.9179128 \\
\hline 0.07 & 1.9869449 & 1.9889064 & 1.9831310 & 1.9852054 & -1.9783801 & -1.9805402 \\
\hline 0.09 & 1.9972387 & 1.9977602 & 1.9961609 & 1.9967569 & -1.9947182 & -1.9953868 \\
\hline 0.20 & 1.9999994 & 1.9999996 & 1.9999988 & 1.9999992 & -1.9999977 & -1.9999983 \\
\hline 0.40 & 1.9999999 & 1.9999999 & 1.9999999 & 1.9999999 & -1.9999999 & -1.9999999 \\
\hline 0.60 & 1.9999999 & 1.9999999 & 1.9999999 & 1.9999999 & -1.9999999 & -1.9999999 \\
\hline 0.80 & 1.9999912 & 1.9999925 & 1.9999953 & 1.9999962 & -1.9999976 & -1.9999981 \\
\hline 0.90 & 1.9958091 & 1.9961390 & 1.9969491 & 1.9972484 & -1.9978114 & -1.9980773 \\
\hline 0.92 & 1.9856103 & 1.9865241 & 1.9888380 & 1.9897228 & -1.9914426 & -1.9922850 \\
\hline 0.94 & 1.9505922 & 1.9529645 & 1.9591622 & 1.9616149 & -1.9665411 & -1.9690430 \\
\hline 0.96 & 1.8303554 & 1.8358300 & 1.8505883 & 1.8566322 & -1.8691769 & -1.8757825 \\
\hline 0.98 & 1.4175147 & 1.4269904 & 1.4533526 & 1.4645230 & -1.4884864 & -1.5015676 \\
\hline 1.00 & 0.0000000 & 0.0000000 & 0.0000000 & 0.0000000 & 0.0000000 & 0.0000000 \\
\hline
\end{tabular}

Table 9. Numerical solution of Example 2 for $\varepsilon=0.01$ and $\delta=0.005$

\begin{tabular}{|c|c|c|c|c|c|c|}
\hline \multirow{2}{*}{$x$} & \multicolumn{2}{|c|}{$\eta=0.000$} & \multicolumn{2}{|c|}{$\eta=0.003$} & \multicolumn{2}{|c|}{$\eta=0.006$} \\
\hline & Num. Sol & Exact Sol. & Num. Sol & Exact Sol. & Num. Sol & Exact Sol. \\
\hline 0.00 & 1.0000000 & 1.0000000 & 1.0000000 & 1.0000000 & 1.0000000 & 1.0000000 \\
\hline 0.01 & 0.4146382 & 0.4297281 & 0.4656946 & 0.4840175 & -0.5171991 & -0.5393302 \\
\hline 0.03 & 1.5572662 & 1.5697885 & 1.5986780 & 1.6128849 & -1.6377519 & -1.6537310 \\
\hline 0.05 & 1.8763606 & 1.8821338 & 1.8950278 & 1.9011479 & -1.9115028 & -1.9179128 \\
\hline 0.07 & 1.9654720 & 1.9677078 & 1.9725428 & 1.9747575 & -1.9783801 & -1.9805402 \\
\hline 0.09 & 1.9903575 & 1.9911528 & 1.9928181 & 1.9935541 & -1.9947182 & -1.9953868 \\
\hline 0.20 & 1.9999913 & 1.9999928 & 1.9999955 & 1.9999964 & -1.9999977 & -1.9999983 \\
\hline 0.40 & 1.9999999 & 1.9999999 & 1.9999999 & 1.9999999 & -1.9999999 & -1.9999999 \\
\hline 0.60 & 1.9999999 & 1.9999999 & 1.9999999 & 1.9999999 & -1.9999999 & -1.9999999 \\
\hline 0.80 & 1.9999994 & 1.9999996 & 1.9999988 & 1.9999991 & -1.9999976 & -1.9999981 \\
\hline 0.90 & 1.9989180 & 1.9991155 & 1.9984514 & 1.9986829 & -1.9978114 & -1.9980773 \\
\hline 0.92 & 1.9951293 & 1.9958546 & 1.9935113 & 1.9942997 & -1.9914426 & -1.9922850 \\
\hline 0.94 & 1.9780746 & 1.9805718 & 1.9728123 & 1.9753294 & -1.9665411 & -1.9690430 \\
\hline 0.96 & 1.9013022 & 1.9089464 & 1.8860824 & 1.8932266 & -1.8691769 & -1.8757825 \\
\hline 0.98 & 1.5557078 & 1.5732598 & 1.5226791 & 1.5378889 & -1.4884864 & -1.5015676 \\
\hline 1.00 & 0.0000000 & 0.0000000 & 0.0000000 & 0.0000000 & 0.0000000 & 0.0000000 \\
\hline
\end{tabular}

\section{References}

[1] R. Bellman, and K. L. Cooke, Differential-Difference Equations. Academic Press, New York, 1963.

[2] R. D. Driver, Ordinary and Delay Differential Equations, Springer-Verlag, New York, 1977.

[3] K. Denevers and K. Schmit, "An application of the shooting method to boundary value problems for second-order delay equations", J. Math. Anal. Appl., 36 (1971) 588-597.

[4] G. B. Gustafson and K. Schmitt, "Nonzero solutions of boundary value problems for second order ordinary and delay differential equations", J. Differential Equations, 12 (1972) 129-147.

[5] R.B. Stein, "A theoretical analysis of neuronal variability", Biophys. J. 5 (1965) 173-194.

[6] H. C. Tuckwell and W. Richter, "Neuronal inter-spike time distributions and the estimation of neuro-physiological and neuroanatomical parameters", J. Theor. Biol., 71 (1978) 167-183.

[7] H. C. Tuckwell and D. K.Cope, "Accuracy of neuronal inter-spike times calculated from a diffusion approximation", J. Theor. Biol., 83 (1980) 377-387.

[8] W. J. Wilbur and J. Rinzel, "An analysis of Stein's model for stochastic neuronal excitation", Biol. Cybern., 45 (1982) 107-114.
[9] C.G. Lange R.M. Miura, "Singular perturbation analysis of boundary-value problems for differential difference equations", SIAM J. Appl. Math., 42 (1982) 502-531.

[10] C.G. Lange R.M. Miura, "Singular perturbation analysis of boundary-value problems for differential-difference equations. V. Small shifts with layer behaviour", SIAM J. Appl. Math., 54 (1994) 249-272.

[11] C.G. Lange R.M. Miura, "Singular perturbation analysis of boundary-value problems for differential-difference equations. VI. Small shifts with rapid oscillations", SIAM J. Appl. Math., 54 (1994) 273-283.

[12] M. K. Kadalbajoo and K. K. Sharma, "Numerical analysis of boundary-value problems for singularly perturbed differentialdifference equations with small shifts of mixed type", J. Optim. Theory Appl., 115 (1) (2002) 145-163.

[13] M. K. Kadalbajoo and K. K. Sharma, "Numerical treatment of a mathematical model arising from a model of neuronal variability", J. Math. Anal. Appl., 307 (2005) 606-627.

[14] L. E. El'sgolts and S. B. Norkin, Introduction to the Theory and Applications of Differential Equations with Deviating Arguments, Academic Press, New York, 1973.

[15] R. K. Mohanty and N. Jha, "A class of variable mesh spline in compression methods for singularly perturbed two point singular boundary value problems", Applied Mathematics and Computation, 168 (2005) 704-716. 